\title{
EXINI Quantitative Bone Scan Index: Expanded Utility for the Planar Radionuclide Bone Scan
}

\author{
Steven M. Larson \\ Memorial Sloan Kettering Cancer Center, New York, New York
}

John Henry was a steel drivin' man. . .

— "The Ballad of John Henry," American folk song

interpreting a bone scan. You carefully inject the bone-seeking radiopharmaceutical, such as $925 \mathrm{MBq}$ of ${ }^{99 \mathrm{~m}} \mathrm{Tc}-\mathrm{methylene}$ diphosphonate, in a good vein; wait $90 \mathrm{~min}$ to let the radioactivity target metabolically active bone; ask the patient to urinate; position the patient comfortably supine on the scanning bed of a dual-head $\gamma$-camera; set the machine to image from head to toe at $8-10 \mathrm{~cm} / \mathrm{min}$; activate "go"; and in about $20 \mathrm{~min}$ the imaging is done and the patient is ready to go home. In the process, you have used tracer radioactivity to create an electronic record-a functional snapshot of living bone, the moment-by-moment biochemistry that is a signature of the metabolic homeostasis of the patient's skeleton. You bring up the simultaneously acquired anterior and posterior planar images and view the results on the electronic workstation. What you see is a usually light gray image of a skeleton (Supplemental Fig. 1; available at http://jnm. snmjournals.org) viewed with high enough resolution to reveal even the smallest bone, in front and back projections. You search for hotspots: sites of increased uptake caused by an increased exchange of radionuclide for bone and bone minerals such as occurs near a metastasis. On the image, a hotspot is superimposed on the normal bony contour. Depending on the pattern of uptake, you assign a probability that the hotspot is actually a site of bony metastatic disease, with important implications for treatment of the patient's cancer. Because of its simplicity and ease of performance, the planar bone scan continues to be widely used in nuclear medicine despite advances in SPECT and PET imaging.

Modern approaches to the visual interpretation of bone scans reproducibility and especially speed, greatly extending the potential for rapid, quantitative analysis of planar bone scans.

The old, reliable planar bone scan has been the bedrock test of nuclear medicine for decades. The right radiometal with calciumlike properties or the appropriate radionuclide chelated with phosphonates can be used to accurately diagnose bony metastases in osteotropic cancers, usually before CT can show them! The pathophysiology of detecting regional metastases involves accelerated hydroxyapatite crystal turnover in the metabolically active bone near the metastatic tumor. It is the "do one, see one, teach one" test of nuclear medicine. By their second day in nuclear medicine (the first day would be a lecture on safe handling of radioactivity and bone scan interpretation and use), even the greenest residents can do a reasonable job of performing and

Received Sep. 22, 2015; revision accepted Sep. 23, 2015.

For correspondence or reprints contact: Steven M. Larson, Memorial Sloan Kettering Cancer Center, 1275 York Ave., S-212, New York, NY 10021.

E-mail: larsons@mskcc.org

Published online Oct. 8, 2015

COPYRIGHT (C) 2016 by the Society of Nuclear Medicine and Molecular Imaging, Inc.

DOI: $10.2967 /$ jnumed.115.164137 are still an important part of nuclear medicine and are used for the clinical indication of suspected metastases in osteotropic tumors such as prostate and breast cancer. We know that even a single verified metastatic lesion in bone greatly modifies prognosis adversely. The planar bone scan has adapted to the age of precision medicine and is selectively used in the management of individual patients, such as in prostate cancer when prostatespecific antigen biomarkers are elevated above $10 \mathrm{ng} / \mathrm{mL}$ (2). Moreover, the value of planar bone scanning has been greatly enhanced by the methodology of the Prostate Cancer Clinical Trials Working Group 2 (PCWG2) for measuring radiographic progression (3). This approach is also based on visual interpretationvisual interpretation of a series of bone scans that serves as a kind of contemporaneous reference, timed with respect to the beginning of treatment, to overcome the effect of the flair phenomenon. The flair phenomenon, in retrospect, creates false-positive results that, in the context of clinical trials, have the bad result of misclassifying drug effects. In contrast, when used according to the adapted PCWG2 radiographic-progression criteria (4), bone scans have been shown to correlate strongly with survival in large trials of the use of enzalutamide and abiraterone in prostate cancer (5). This 
approach to radiographic progression assisted in identifying a positive effect on advanced prostate cancer and has been accepted by the U.S. Food and Drug Administration as a parameter with a potential impact on drug approval decisions (6).

I am happy to say that the EXINI approach (EXINI Diagnostics $\mathrm{AB}$ ) is a neural network-based computerized extension of a manual method- the BSI method for quantitating metastatic disease on planar bone scans-developed by our team of collaborators at Memorial Sloan Kettering Cancer Center in the late 1980s $(7,8)$. At that time, we became interested in the quantitative potential of bone scans and developed the BSI technique to measure the fraction of total skeleton based on subjective interpretation of bone-by-bone involvement using bone weights from reference man. In our manual approach we created a list of all bones, and their percentage contribution to the total skeleton, and estimated fractional bony involvement by adding each positive bone to obtain a BSI or percentage of tumor-involved skeleton. We introduced this method as a research tool primarily for prostate cancer staging. We showed that BSI was strongly prognostic, with higher numbers correlating with worse prognosis, and that with simple training there was good intraobserver and interobserver agreement. We applied this tool in numerous clinical trials and saw encouraging results $(9,10)$. The biggest difficulty was the effort and time required to accurately estimate the BSI, especially in advanced disease. It took a fellow or technician literally several weeks to analyze a trial of more than 200 bone scans, and that effort was too labor-intensive to be practical.

Other applications of visual interpretation of planar bone scanning include going beyond prognosis to evaluate biologic features, as shown by the correspondence between early metastasis in bone and the distribution of active adult red marrow (7). Additional evidence indicates that bone marrow involvement as measured by ${ }^{18} \mathrm{~F}$-FDG PET precedes positive bone scan results by about $6-8$ wk (11). As an example of pathobiology that can be studied with the BSI, we used the manual BSI to evaluate the rate at which a change in bony metastases occurred in individual patients with prostate cancer. We found that in a group of patients evaluated with sequential bone scanning, the rate of progression by metastatic change followed Gompertzian kinetics (Supplemental Fig. 2). If we track these patients back to the time of a very low BSI ( $<1 \% ; 27$ patients) and plot the site of their initial lesions, we find that the site matches the distribution of red or active bone marrow quite precisely.

When Edenbradt et al. developed EXINI as a computerized approach (12), I was hopeful that a rapid method had been found. However, the initial variations in methodology, although precise and reproducible, broke down at higher BSIs of more than $10 \%$ and gave much lower numbers than the manual BSI method, suggesting the possibility of inaccuracy in total skeletal estimates.

In the current paper, the EXINI group reports a revised computer model that corrects these deficiencies and-although effectiveness in large datasets still needs to be documentedappears to open the door to rapid analysis of the BSI. If successful, the new computerized method could be used in principle for clinical trials involving thousands of patients, applying a common standardized parameter for treatment response that will allow for quantitative, objective stratification of patients and analysis of flair and progression. Finally, a computerized method will be highly valuable in standardizing the use of planar bone scanning according to PCWG2 criteria in advanced prostate cancer (Supplemental Fig. 3) and perhaps in bone-only breast cancer. The precision of these methods is outstanding, and the EXINI BSI might also be extended to 3-dimensional methodologies, which has not been done for our manual BSI method because of the labor involved and the multiple images required.

The improved EXINI BSI comes at an ideal time, when novel drugs have extended the life of patients with bony metastases and greater monitoring of treatment response is required. Moreover, these quantitative computer-based algorithms are fundamentally applicable to any bone-seeking radiopharmaceutical.

\section{DISCLOSURE}

No potential conflict of interest relevant to this article was reported.

\section{REFERENCES}

1. Anand A, Morris MJ, Kaboteh R, et al. Analytic validation of the automated bone scan index as an imaging biomarker to standardize quantitative changes in bone scans of patients with metastatic prostate cancer. J Nucl Med. 2016;57:41-45.

2. Larson SM. Prostate cancer: better use of bone scans in prostate cancer. Nat Rev Urol. 2015;12:190-191.

3. Scher HI, Halabi S, Tannock I, et al. Design and end points of clinical trials for patients with progressive prostate cancer and castrate levels of testosterone: recommendations of the Prostate Cancer Clinical Trials Working Group. J Clin Oncol. 2008;26:1148-1159.

4. Morris MJ, Halabi S, Tannock I, et al. Radiographic progression-free survival as a response biomarker in metastatic castration-resistant prostate cancer: COUAA-302 results. J Clin Oncol. 2015;33:1356-1363.

5. Ryan CJ, Smith MR, de Bono JS, et al. Abiraterone in metastatic prostate cancer without previous chemotherapy. N Engl J Med. 2013;368:138-148.

6. Kluetz PG, Ning YM, Maher VE, et al. Abiraterone acetate in combination with prednisone for the treatment of patients with metastatic castration-resistant prostate cancer: U.S. Food and Drug Administration drug approval summary. Clin Cancer Res. 2013;19:6650-6656.

7. Imbriaco M, Larson SM, Yeung HW, et al. A new parameter for measuring metastatic bone involvement by prostate cancer: the bone scan index. Clin Cancer Res. 1998;4:1765-1772.

8. Sabbatini P, Larson SM, Kremer A, et al. Prognostic significance of extent of disease in bone in patients with androgen-independent prostate cancer. $J$ Clin Oncol. 1999;17:948-957.

9. Dennis ER, Jia X, Mezheritskiy IS, et al. Bone scan index: a quantitative treatment response biomarker for castration-resistant metastatic prostate cancer. $J$ Clin Oncol. 2012;30:519-524.

10. Scher HI, Morris MJ, Larson S, Heller G. Validation and clinical utility of prostate cancer biomarkers. Nat Rev Clin Oncol. 2013;10:225-234.

11. Meirelles GS, Schöder H, Ravizzini GC, et al. Prognostic value of baseline $\left[{ }^{18} \mathrm{~F}\right]$ fluorodeoxyglucose positron emission tomography and ${ }^{99 \mathrm{~m}} \mathrm{Tc}-\mathrm{MDP}$ bone scan in progressing metastatic prostate cancer. Clin Cancer Res. 2010;16:6093-6099.

12. Edenbradt. 